\title{
Pyomyositis of Obturator Muscles: Unusual Late Presentation
}

\author{
Prasad Channappa Soraganvi ${ }^{1}$, Ramakanth $\mathrm{R}^{1}$
}

\author{
What to Learn from this Article? \\ Presentation of Pyomyositis of Obturator muscles and how to diagnose it. \\ Management of Obturator Pyomyositis and expected results.
}

Abstract

Introduction: Pyomyositis of obturator muscles is rare condition. Late presentation with deformities of hip misleads the clinician. Late presentation ( 6 weeks) of this condition has not been reported earlier. This report highlights this unusual presentation of Pyomyositis of the obturator muscles.

Case Report: We are reporting a 14year old female patient presented with limp and pain in hip since 6 weeks. Her hip radiographs were unremarkable. Patient was admitted and MRI done. MRI findings were consistent with obturator pyomyositis. Diagnosis of pyomyositis confirmed by MRI and we performed percutaneous aspiration and drained about $25 \mathrm{ml}$ of purulent material mixed with blood. The culture grew Staphylococcus aureus. Patient received intravenous antibiotic for 1 week and oral antibiotic for 2 weeks. Patient was immobilized in fixed skin traction in Thomas splint for 5days, later gentle mobilization was started. Her condition improved dramatically after aspiration. A follow up MRI done at 3 weeks following aspiration revealed a significant reduction in intramuscular collection of obturator internus and obturator externus. Three weeks following aspiration patient was relieved of the pain and was able to walk normally. At 6 months follow up visit patient was asymptomatic.

Conclusion: Late presentation of obturator pyomyositis is rare. We emphasise on careful examination and need for early imaging for diagnosis. Percutaneous drainage results in successful treatment.

Keywords: Pyomyositis, septic arthritis, infection, obturator muscle.

\section{Introduction}

Author's Photo Gallery

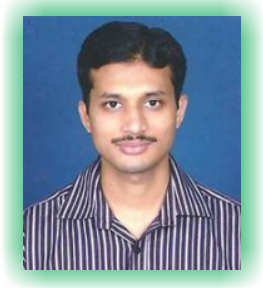

Dr. Prasad Soraganvi

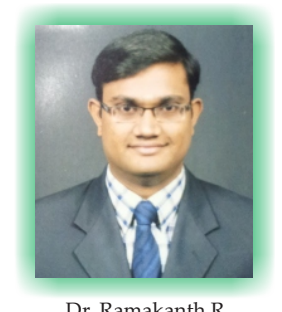

Dr. Ramakanth R

Pyomyositis of the obturator muscles is an uncommon entity often mistaken for septic arthritis of the hip.[1] Lack of familiarity with this entity frequently leads to a delayed diagnosis or misdiagnosis. Often in a developing countries like India patients presents late and were previously treated empirically without a firm diagnosis. To date all the cases of Primary Pyomyositis of the obturator muscles have been reported at early presentation (three weeks) with fever, sepsis, hip pain, inability to bear weight and painful limitation of movements. We report the case which is presented late (six weeks) with confusing array of findings. The correct diagnosis can be difficult unless the pyomyositis is kept as a differential diagnosis.

${ }^{1}$ PESIMSR

Medical college, Kuppam, Andhra Pradesh, India.

Address of Correspondence

Dr Prasad Channappa Soraganvi

Medical college, Kuppam, Andhra Pradesh, India.

Email: prasad_doct@yahoo.co.in

\section{Case Report}

A 14 year old girl presented with pain in the left hip, pyrexia and difficulty in walking since six weeks. Initially hip pain was dull aching and exacerbated on walking. Over next six days dull aching pain progressively increased to more severe pain and patient developed difficulty in walking. 


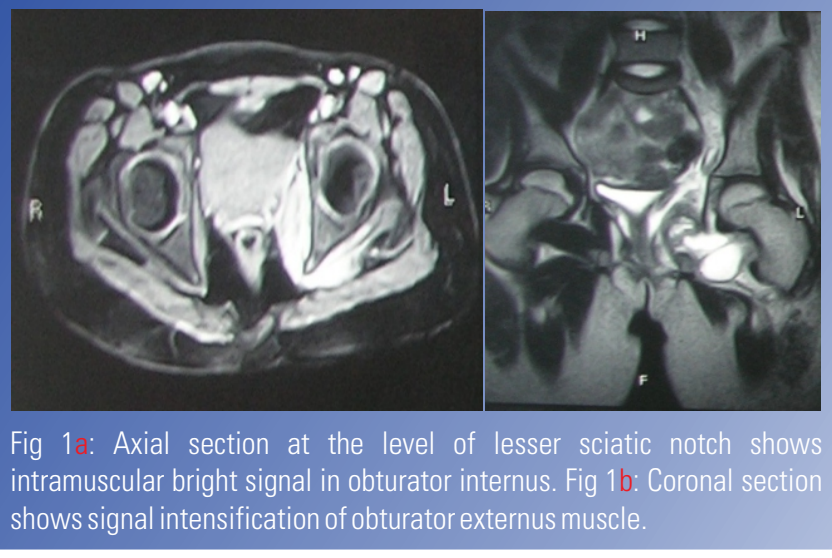

Fever started one day after hip pain which was low grade and intermittent. There was no history of any fall or trauma. Initially patient was treated by different clinician with oral analgesics and antibiotics for seven days but there was no improvement.

At the time of admission patient had temperature of $39^{\circ} \mathrm{C}$ and pulse of 98 per minute. Left lower limb was in attitude of flexion, abduction and external rotation. Local warmth and tenderness was present in left scarpa's triangle and gluteal area. She had restriction of movements with fixed flexion deformity of $20^{\circ}$ with further free flexion of $30^{\circ}$ possible, fixed abduction deformity of $25^{\circ}$ with further free abduction of $20^{\circ}$, and fixed external rotation deformity of $10^{\circ}$ with free external rotation of $20^{\circ}$ possible beyond which movements were pain full. She had $2 \mathrm{~cm}$ apparent shortening due to her fixed deformities but there was no true shortening. Ipsilateral knee, contra lateral hip, knee and ankle were clinically normal

Initial laboratory studies revealed ESR $125 \mathrm{~mm} / \mathrm{hr}$, white blood cell count 11,100 cells/cu.mm with polymorphs $61 \%$ lymphocytes 30\%. An anteroposterior (AP) radiograph of both hips and lateral radiograph of left hip was unremarkable. She was admitted and MRI was performed on following day. MRI demonstrated (fig 1a and $1 \mathrm{~b}$ ) altered signal intensity of obturator internus and obturator externus muscles of left side with intramuscular collection. There was no effusion in joints and no obvious demonstrable pathology noted in articular cartilage.

Pyomyositis of obturator muscles was confirmed on MRI findings. We performed percutaneous aspiration following day and drained about $25 \mathrm{ml}$ of purulent material mixed with blood. Sample of pus was sent for microbiological investigation. Patient started on 1rd generation cephalosporin (tab. Cephalexin) antibiotic initially. The culture grew Staphylococcus aureus. Later based on antibiotic sensitivity report, Augmentin intravenous antibiotic (Amoxycillin and Clavulanic acid)

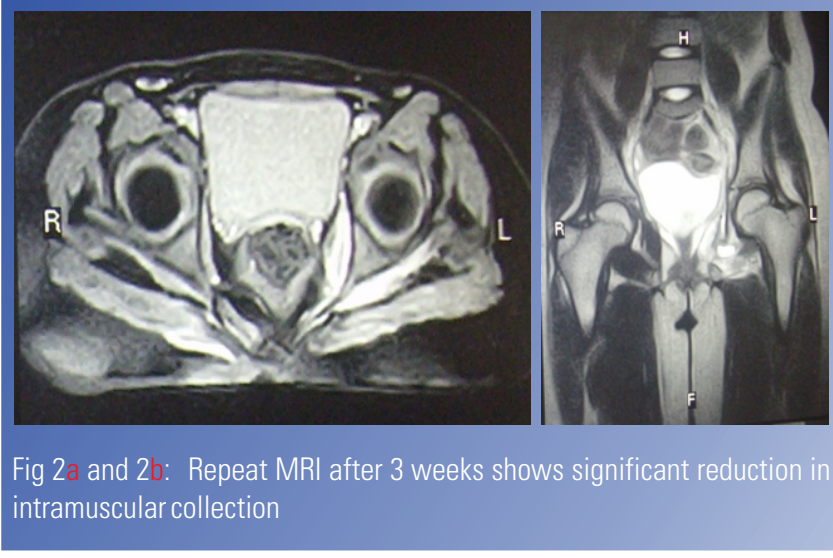

was given for 1 week and oral antibiotics continued for two weeks. Patient was immobilized in fixed traction in Thomas splint for 5 days and then gentle mobilization was started. Her condition improved dramatically after aspiration and she was discharged after 7 days. Postoperative recovery was satisfactory with increase in range of movement and correction of fixed deformities. At 3 weeks follow up visit patient was asymptomatic and was ambulating normally. Repeat MRI at 3 weeks revealed a significant reduction in intramuscular collection of obturator internus and obturator externus (fig $2 \mathrm{a}$ and fig 2b). At 6 months follow up patient was asymptomatic.

\section{Discussion}

The obturator internus muscle originates from the anterolateral wall of the pelvic cavity around the obturator foramen and it exits the pelvic cavity through the lesser sciatic foramen and inserts into the greater trochanter along with the superior and inferior gemelli. It abducts and externally rotates the hip joint. Pyomyositis is a primary, sub acute and deep bacterial infection of the skeletal muscle that manifests itself as localized abscess formation, a diffuse inflammatory or a rapidly progressing myonecrotic process. [1]

Obturator muscle pyomyositis is a rare entity, [2,3] and is often not a principal diagnosis, as it shares the same symptoms and signs of the more alarming and common septic arthritis. In a review of 14 cases of obturator pyomyositis, the common manifestations noted were hip or thigh pain, fever and sepsis and limp. On clinical examination majority of patients had the involved hip in a flexed, abducted and externally rotated. [4] This clinical picture frequently leads to misdiagnosis such as septic arthritis of hip causing severe morbidity. [1,5]

There are, however, a number of distinguishing features. Obturator pyomyositis does not reduce hip movement so dramatically, and is associated with more discrete areas of 
tenderness, including pain on rectal examination when the obturator internus is involved. There may be pain radiating to the leg if the sciatic nerve is involved, $[3,6]$ and in females, there may be oedema of the ipsilateral labia.[2,7] Although these distinguishing features may suggest diagnosis at early presentation, but in late presentation like our case these may not be helpful.

Local trauma is a recognised initiating factor for pyomyositis.[3] It is documented in between $21 \%$ and $66 \%$ of cases. [8,9] Local trauma to the muscle that results in inflammation or haematoma is thought to be important in addition to concurrent episode of bacteraemia.[10,11] Our patient did not give any history of trauma preceding initial symptoms.

The causative organism is usually Staph. aureus $(81 \%),[12]$ the rest were Streptococcus pyogenes and Neisseria gonorrhoea. [13] Others have documented similar bacteriological profiles for pyomyositis in general.[8,9] Involvement of the obturator muscles has also been reported with $\mathrm{N}$. gonorrhoea in women but it seems likely that these were secondary to local pelvic spread.[3] As these organisms commonly cause bacteraemia in children, any initial empirical antibiotic therapy being considered should cover the abovementioned organisms. [2,3]

In a majority of patients, treatment with appropriate antibiotics alone is sufficient.[14] Surgical drainage is rarely indicated if the patient fails to respond to medical therapy with unusual organism. [14] Antibiotics of choice should be efficient against S. aureus. The duration of antibiotics has not been established and can vary from 2 to 6 weeks, depending on the clinical severity and response to antibiotics. $[3,15]$ Our patient received empirical oral antibiotic initially for 1 week, which was for short duration and infection was still persisting. Hence our patient manifested at 6 weeks pain, low grade fever and fixed deformities.

However, when medical management is unsuccessful, as demonstrated by persistence of fever pain for more than 5 to 7 days (particularly in cases where blood cultures are sterile), percutaneous guided drainage is indicated. [2] Surgical drainage should be reserved for cases of obturator abscess complicated by osteomyelitis because the anatomy at the site of the obturator muscle renders the percutaneous drainage hazardous. [2] In a review of 22 cases by R.J.King et al. 54\% of cases the infection resolved with intravenous antibiotic treatment alone. In five patients $(23 \%)$ percutaneous aspiration was performed, and in another five (23\%), incision and drainage were required. [12] We performed percutaneous aspiration for our case, as the presentation was late and initial antibiotic treatment was given for short duration.

In a review of 22 cases as reported by R.J.King et al. [12] shows all reported cases of pyomyositis of obturator muscles had presented within three weeks of initial symptoms, however this is first reported case which presented at six weeks with limp and fixed deformities of hip. Most of the cases reported were within one weeks of initial symptom and very few at 3 weeks. The case presented here highlights unusual late presentation (at 6 weeks) of obturator pyomyositis which itself is rare. This patient presented with difficulty in walking and fixed deformities of hip. Pain and fever was present but it was of mild grade. Most of reported cases had pain and fever with toxic symptoms as presenting complaint which was absent in the case reported here. This clinical picture created more difficulty in diagnosis, as tuberculosis of hip is common in India and presents with similar features which made us to misdiagnose this case as tuberculosis of hip initially. In our patient MRI done following day of admission and MRI findings were consistent with pyomyositis of obturator muscle. Percutaneous aspiration with antibiotic cover leads complete resolution.

\section{Conclusion}

Late presentation of obturator pyomyositis is rare; diagnosis depends on careful examination and early use of magnetic resonance imaging. Percutaneous drainage results in successful treatment.

\section{Clinical Message}

Late presentation of obturator pyomyositis is diagnostic challenge to the clinician as the patients present with unusual pain around hip joint with pyrexia and fixed deformities. Careful history, clinical examination and MRI needed for diagnosis and can be successfully treated by percutaneous drainage

\section{References}

1.Orlicek SL, Abramson JS, Woods CR,Givner LB. Obturator internus muscle abscess in children. J Pediatr Orthop. 2001;21:7448.

2.Viani RM, Bromberg K, Bradley JS. Obturator internus muscle abscess in children: report of seven cases and review. Clin Infect Dis. 1999;28:117-22.

3. Birkbeck D, Watson JT. Obturator internus pyomyositis. A case report. Clin Orthop Relat Res. 1995;316:221-6.

4. Wong RKF,1 Ng BWK,1 Greg A2. A Rare Condition Mimicking 
Septic Hip in Children-Case Report of a Child with Obturator Internus Muscle Pyomyositis. Hong Kong J Orthop Surg.2006;10(1):39-41.

5. Nikolopoulos DD, Apostolopoulos A, Polyzois I, Liarokapis S, Michos I. Obturator internus pyomyositis in a young adult: a case report and review of the literature. Cases J. 2009;2:8588.

6. Chatwani A, Shapiro T, Mitra A, Levtoaff A, Reece EA. Postpartum paravaginal hematoma and lower-extremity infection. Am JObstet Gynecol 1992;166:598-600.

7. Guis-Sabatier S, Pieri-Balandraud N, Garnier-Soumet P, et al. Pubic pain in athletes: a case due to an abscess in the obturator muscle. Rhum EnglEd 1999;66:58-60.

8. Chacha PB. Muscle abscesses in children. Clin Orthop 1970;70: 174-80.

9. Chiedozi LC. Pyomyositis: review of 205 cases in 112 patients. Am
J Surg 1979; 137:255-9.

10. Schlech WF, Moulton P, Kaiser AB. Pyomyositis: tropical disease in a. temperate climate. Am JMed 1981;71:900-2.

11.Echeverria P, Vaughn C. "Tropical pyomyositis." A diagnostic problem S. aureus- in temperate climates. Am J Dis Child 1975;129:856-7.

12. R. J. King, D. Laugharne, R. W. Kerslake, B. J. Holdsworth. Primary obturator pyomyositis: a diagnostic challenge. J Bone Joint Surg [Br] 2003;85-B:895-8.

13.Grose C. Staphylococcal pyomyositis in south Texas. J Pediatr. 1978:93:457-8.

14.Bansal M, Bhaliak V, Bruce CE. Obturator internus muscle abscess in a child: a case report. J Pediatr Orthop B. 2008;17:223-4. .

15. Christin L,Sarosi GA. Pyomyositis in North America: case reports and review. Clin Infect Dis. 1992;15:668-77.
Conflict of Interest: Nil

Source of Support: None

\section{How to Cite this Article:}

Soraganvi PC, Ramakanth R. Pyomyositis of obturator muscle: unusual late presentation.

Journal of Orthopaedic Case Reports 2013 July-Sep;3(3):7-10 